\title{
Characterization of novel IncRNAs in upper thoracic spinal cords of rats with myocardial ischemia-reperfusion injuries
}

\author{
ZHI-XIAO LI $^{1 *}$, YU-JUAN LI ${ }^{1 *}$, QIAN WANG ${ }^{1}$, ZHI-GANG HE $^{2}$, MAO-HUI FENG ${ }^{3,4}$ and HONG-BING XIANG ${ }^{1}$ \\ Departments of ${ }^{1}$ Anesthesiology and Pain Medicine, and ${ }^{2}$ Emergency Medicine, Tongji Hospital, Tongji Medical College, \\ Huazhong University of Science and Technology, Wuhan, Hubei 430030; ${ }^{3}$ Department of Gastrointestinal Surgery, \\ Zhongnan Hospital, Wuhan University; ${ }^{4}$ The Clinical Medical Research Center of Peritoneal Cancer of Wuhan, \\ Clinical Cancer Study Center of Hubei Provence, Key Laboratory of Tumor Biological Behavior of Hubei Provence, \\ Wuhan, Hubei 430071, P.R. China
}

Received November 13, 2019; Accepted August 19, 2020

DOI: 10.3892/etm.2021.9783

\begin{abstract}
Myocardial ischemia-reperfusion injury (MIRI) is a significant problem in clinical cardiology, and refers to a more serious myocardial injury caused by blood recanalization after a period of myocardial ischemia, as compared with injury caused by vascular occlusion. The spinal cord, as the primary afferent and efferent center of cardiac sensory and sympathetic nerve fibres, has received increased attention in recent years with regards to the regulation of MIRIs. Previous studies have revealed that MIRI has a strong correlation with the abnormal expression of long non-coding (lnc)RNAs in the myocardium; however, there are limited reports on the effects of the altered expression of lncRNAs in the spinal cord following MIRI. To investigate the expression patterns of lncRNAs in the spinal cord after MIRI and their potential role in the early stage of reperfusion, a MIRI model was established in rats. After $30 \mathrm{~min}$ of myocardial ischemia and $2 \mathrm{~h}$ of reperfusion, the upper thoracic spinal cord tissues were immediately dissected and isolated. lncRNAs and mRNAs in spinal cord tissues were screened using transcriptome sequencing technology, and the expression of several highly deregulated mRNAs, including Frs3, Zfp52, Dnajc6, Nedd41, Tep1, Myef2, Tgfbr1, Fgf12, Mef2c, Tfdp1 and lncRNA, including
\end{abstract}

Correspondence to: Professor Mao-Hui Feng, Department of Gastrointestinal Surgery, Zhongnan Hospital, Wuhan University, 169 Donghu Road, Wuhan Hubei 430071, P.R. China

E-mail: fengmh5690@163.com

Professor Hong-Bing Xiang, Department of Anesthesiology and Pain Medicine, Tongji Hospital, Tongji Medical College, Huazhong University of Science and Technology, 1095 Jiefang Avenue, Wuhan, Hubei 430030, P.R. China

E-mail: xhbtj2004@163.com

*Contributed equally

Key words: myocardial ischemia-reperfusion injury, long non-coding RNA, mRNA, spinal cord
ENSRNOT00000080713, ENSRNOT00000090564, ENSRNOT00000082588, ENSRNOT00000091080, ENSRNOT00000091570, ENSRNOT00000087777, ENSRNOT00000082061, ENSRNOT00000091108, ENSRNOT00000087028, ENSRNOT00000086475, were further validated via reverse transcription-quantitative PCR. The number of altered expressed lncRNAs was 126, among which there were 41 upregulated probe sets and 85 downregulated sets. A total of 470 mRNAs were differentially expressed, in which 231 probe sets were upregulated and 239 were downregulated. Gene Ontology analysis indicated that dysregulated transcripts related to biological processes were mainly associated with 'cell-cell signaling'. Moreover, pathway analysis demonstrated significant changes in the 'PI3K/Akt signaling pathway' and the 'p53 signaling pathway'. Thus, the altered expression of IncRNAs in the spinal cord may be of considerable importance in the process of MIRI. The present results could provide an insight into the potential roles and mechanism of lncRNAs during the early stage of reperfusion.

\section{Introduction}

Ischemic heart disease (IHD) is a leading cause of premature mortality worldwide $(1,2)$. IHD affects $\sim 126$ million individuals globally $(1,655$ per 100,000$)$, which is estimated to be $1.72 \%$ of the world's population (1). Restoration of coronary artery perfusion is currently one of the main treatment options but it can lead to myocardial ischemia-reperfusion injury (MIRI), which refers to a more serious myocardial injury caused by blood recanalization after a period of myocardial ischemia compared with that induced by vascular occlusion $(3,4)$. MIRI is reported to increase the risk of cardiovascular disease, which imposes health, social and human burdens on societies worldwide, and thus is a public health issue (5-8). Therefore, treatment of MIRI is important for the prevention of cardiovascular disease, the end stage of cardiac dysfunction and mortality.

Previous studies have reported that the spinal cord is closely associated with the occurrence and development of MIRI (9-11). Following cardiac ischemia and reperfusion, chemical mediators such as dynorphin and suppresses 
substance $\mathrm{P}$ within the myocardium activate cardiac afferent fibres projecting from the dorsal root ganglion to the upper thoracic dorsal horn of the spinal cord. This results in the release of inflammatory factors and neuropeptides within the spinal cord (12-15), which triggers abnormal changes in gene transcription and translation within the spinal cord (16-18). Despite these recognized spinal responses, the genomic mechanisms that enable differences during these spinal processes of MIRI remain largely elusive.

It has been revealed that long non-coding (lnc)RNAs serve a key role in the processing of gene expression signals via the regulation of cell cycle distribution, stem cell differentiation and transcriptional and post-transcriptional control $(19,20)$. Moreover, there are aberrant expression levels of spinal lncRNAs in peripheral diseases, such as pruritus $(21,22)$, acute kidney injury (23), neuropathic pain and inflammation-related pain (24).

In the present study, the altered expression of mRNAs and lncRNAs in the thoracic (T)1-T4 spinal cord were analyzed using high-throughput RNA-sequencing (RNA-seq) between a group of rats established with MIRI and a sham group. Using microarrays, the current study identified 470 mRNAs and 126 lncRNAs that were significantly differentially expressed in rats with MIRI, and further analyzed the biologic roles of these mRNAs and lncRNAs by performing Gene Ontology (GO) and pathway analyses. Subsequently, the differentially expressed mRNAs and lncRNAs were validated using reverse transcription-quantitative (RT-q) PCR. The present results may facilitate the development of molecular-targeting therapies for MIRI.

\section{Materials and methods}

Animals. In total, 26 male Sprague Dawley rats (specific pathogen free grade; weight, 250-300 g; age, 8-10 weeks) from Hunan Slack Jingda Experimental Animal Company were used in the present study. The animals were housed at a controlled room temperature $\left(25 \pm 0.5^{\circ} \mathrm{C}\right)$ and relative humidity $(40-60 \%)$ with a 12-h light/dark cycle and had unlimited access to food and water. The number of animals in each group was three, and each animal was used only once. All experiments were performed with the approval of the Institutional Animal Care and Use Committee of Tongji Hospital, Huazhong University of Science and Technology (IRB ID, TJ-A0804) and the National Institutes of Health Guide for the Care and Use of Laboratory Animals (25).

MIRI. Rats were randomly divided into MIRI (model, $\mathrm{n}=13$ ) and sham (control, $\mathrm{n}=13$ ) groups. MIRI experiments were performed according to previously published protocols $(18,26-29)$. The rats were maintained in deep anesthetic state on a heating pad using sodium pentobarbital $(50 \mathrm{mg} / \mathrm{kg}$; intraperitoneal), which was indicated by disappearance of the foot withdrawal reflex.

In the model group, the left anterior descending coronary artery (LAD) was ligated $2-\mathrm{mm}$ below the left atrial appendage for $30 \mathrm{~min}$ and then reperfused for $2 \mathrm{~h}$, while sutures were placed without LAD ligation in control operations. The reperfused rats were considered as the animal model of MIRI. Sham-operated age-matched rats served as controls. During the experiment, the electrocardiogram (ECG) of animals was monitored using a electrocardiogram machine (model no. ECG-2303B; Guangzhou Sanrui Electronic Technology Co., Ltd.) at the following time points: Before the operation, ischemia for 0,15 and $30 \mathrm{~min}$, reperfusion for $0 \mathrm{~min}$, $30 \mathrm{~min}$ and $2 \mathrm{~h}$. After reperfusion for $2 \mathrm{~h}, 150 \mathrm{mg} / \mathrm{kg}$ sodium pentobarbital via intraperitoneal injection was used for euthanasia and the confirmation of euthanasia was decapitation. Then, the hearts were collected for 2,3,5-triphenyltetrazolium chloride (TTC) staining, hematoxylin and eosin (H\&E) staining and Masson staining. At the end of reperfusion, the rats were deeply anesthetized, blood $(\sim 1 \mathrm{ml})$ samples were collected from the aorta for cardiac troponin I (cTnI) detection before euthanasia.

In the present experiment, $20 \%$ of the animals died due to hemorrhage and arrhythmia caused by ligation of coronary artery at the early stage of reperfusion. When ventricular fibrillation and bleeding were uncontrollable, euthanasia was performed on animals as humane endpoints. Time points of $30 \mathrm{~min}$ for ischemia and $2 \mathrm{~h}$ for reperfusion were selected for this animal model is sufficient to result in significant histopathological and functional myocardial injury, which can be confirmed using the results of ECG, cTnI, TTC, H\&E and Masson staining (30).

cTnI detection and myocardial tissue staining. cTnI level was determined using an Automated Blood or Urine Chemical Analyzer (Vitro 350; Ortho Clinical Diagnostics). $\mathrm{H} \& \mathrm{E}$ staining and Masson trichrome staining were conducted in the myocardial tissue samples in order to observe the myocardial pathology. Heart tissues were fixed in $10 \%$ formaldehyde for $24 \mathrm{~h}$ at room temperature and embedded with paraffin, before $4-\mu \mathrm{m}$ thick sections were cut. After deparaffinization by washing with xylene followed by rehydration in a descending ethanol gradient, sections were immersed in hematoxylin for 5-7 min, differentiated in 1\% acid alcohol for $2-5 \mathrm{sec}$ and stained with $0.5 \%$ eosin for $2 \mathrm{~min}$. After rinsing with distilled water for $30 \mathrm{sec}$, sections were dehydrated with an ascending ethanol gradient and were cleared in xylene. All procedures were carried out at room temperature. Using a light microscope (magnification x200; Leica DM 4000B; Leica Microsystems, Inc.), pathological changes of myocardial tissues were observed. The procedure of Masson's trichrome staining was similar to H\&E staining, which was described previously (31).

The infarct size was assessed using TTC staining $2 \mathrm{~h}$ after reperfusion. After surgery, the hearts were removed and frozen for $20 \mathrm{~min}$ at $-20^{\circ} \mathrm{C}$, then transversally cut into sections with a thickness of 1-2 mm. Tissue sections were incubated in $2 \%$ TTC for $10 \mathrm{~min}$ at $37^{\circ} \mathrm{C}$ in dark conditions and then fixed in $10 \%$ formaldehyde at $4^{\circ} \mathrm{C}$ overnight. Under light microscopic observation, the infarct area was a white color, and the healthy tissues had a red color. The degree of myocardial infarction was represented by the percentage of infarcted area accounting for the left ventricle.

Tissue extraction and RNA isolation. In total, three rats in the MIRI group and three from the sham group were anesthetized, and the T1-T4 spinal cord segments were quickly extracted. Total RNAs were collected from the dorsal horn of the spinal 
cord using TRIzol ${ }^{\circledR}$ reagent (Invitrogen; Thermo Fisher Scientific, Inc.) based on the manufacturer's instructions. The RNA concentration and purity were examined using a spectrophotometer (Invitrogen; Thermo Fisher Scientific, Inc.) $(32,33)$. Equal amounts of mRNA $(1 \mu \mathrm{g})$ from one rat with the same treatment was mixed as one sample after these tests. A total of six samples were selected for microarray analysis.

Gene expression analysis. High-throughput lncRNA-seq was used to identify novel genes in the spinal cord that regulate cardiac function in the rat MIRI model. Briefly, total RNA was isolated from the T1-T4 of spinal cord tissues from the three MIRI- and sham-group rats, and the concentration, purity and integrity of the RNA were assessed on an Agilent 2100 Bioanalyzer (Agilent Technologies, Inc.) and checked using RNase-free $1 \%$ agarose gel electrophoresis containing GelRed $^{\circledR}$ (cat. no. 41003; Biotium, Inc.). Ribosomal RNA was then removed from the total RNA. The mRNA and ncRNA were mostly retained and the strand-specific library was then constructed using NEBNext ${ }^{\circledR}$ Ultra $^{\mathrm{TM}}$ RNA Library Prep Kit for Illumina ${ }^{\circledR}$ (cat. no. E7530L; New England BioLabs, Inc.) according to the manufacturer's protocols. Briefly, the mRNA was fragmented and used as a template to synthesize cDNA with random primers. The first strand cDNA was synthesized using the following progress: $25^{\circ} \mathrm{C}$ for $10 \mathrm{~min}, 42^{\circ} \mathrm{C}$ for $15 \mathrm{~min}, 70^{\circ} \mathrm{C}$ for $15 \mathrm{~min}$ and final holding at $4^{\circ} \mathrm{C}$. A Second Strand Marking Master Mix was used to synthesize second strand cDNA at $16^{\circ} \mathrm{C}$ for $60 \mathrm{~min}$. After using a ligate adapter, uracil-N-glycosylase treatment and PCR amplification were used to construct a sequencing library. The loading concentration of DNA was $12.182 \mathrm{nM}$. The PCR thermocycling parameters were set as follows: Initial denaturation at $98^{\circ} \mathrm{C}$ for $30 \mathrm{sec}$; followed by 15 cycles of $98^{\circ} \mathrm{C}$ for $10 \mathrm{sec}, 60^{\circ} \mathrm{C}$ for $30 \mathrm{sec}$ and $72^{\circ} \mathrm{C}$ for $30 \mathrm{sec} ; 1 \mathrm{cycle}$ at $72^{\circ} \mathrm{C}$ for $5 \mathrm{~min}$ and final holding at $10^{\circ} \mathrm{C}$. The library was then sequenced by Illumina HiSeqÔ 4000 with pair ends reads of $150 \mathrm{bp}$. The data were analyzed by Gene Denovo Biotechnology Co. (https://www. genedenovo.com; Guangzhou, China). The differentially expressed mRNAs and IncRNAs were identified with $\log _{2}$ fold change (FC) $\mid$ and $\mathrm{P}$-values as calculated with a t-test. $\mid \log _{2} \mathrm{FCl}>1$ and $\mathrm{P}<0.05$ were set as the up- and downregulated gene thresholds. Hierarchical clustering and volcano plots determined the different expression patterns of mRNAs and lncRNAs among the samples.

Bioinformatics analysis. The differentially expressed lncRNAs and mRNAs with statistical significance were identified via volcano plot filtering. The threshold used to screen RNAs with $\mid \log _{2} \mathrm{FCl}>1$ was $\mathrm{P}<0.05$. $\mid \log _{2} \mathrm{FCl}>1$ is to screen genes with multiples of difference $>2$ times $(34,35)$. Gene Ontology (GO) analysis (http://www.geneontology.org) described the molecular function, cellular component and biological process of differentially expressed transcripts. GO analysis and a Kyoto Encyclopedia of Genes and Genomes (KEGG) analysis were applied to determine the biologic roles of these differentially expressed lncRNAs and mRNAs based on the latest KEGG data (http://www.genome.jp/kegg/).

RT-qPCR analysis for upper thoracic spinal cord. The total RNA, extracted from the upper T1-T4 segments of the spinal cord using the TRIzol ${ }^{\circledR}$ reagent (Invitrogen; Thermo Fisher Scientific, Inc.) according to the manufacturer's instructions, was used for the generation of cDNA (21,22,36-40). The primers were designed using the Primer Express 3.0 software (Applied Biosystems; Thermo Fisher Scientific, Inc.) and the specific forward and reverse primer sequences are listed in Table I. RNA samples were quantified using a spectrophotometer (BioPhotometer; Eppendorf AG) and then synthesized to cDNA by reverse transcription using the PrimeScript $^{\mathrm{TM}}$ RT reagent kit (Takara Bio, Inc.). The temperature protocol was conducted using the following protocol: $15 \mathrm{~min}$ at $37^{\circ} \mathrm{C}, 5 \mathrm{sec}$ at $85^{\circ} \mathrm{C}$ and holding at $4^{\circ} \mathrm{C} \mathrm{cDNA}$ was quantitated via RT-qPCR using a TB green ${ }^{\circledR}$ Premix Ex Taq (cat. no. RR420A; Takara Bio, Inc.). The thermocycling conditions for PCR were as follows: Initial denaturation for $30 \mathrm{sec}$ at $95^{\circ} \mathrm{C}$, followed by 40 cycles of $15 \mathrm{sec}$ at $95^{\circ} \mathrm{C}, 15 \mathrm{sec}$ at $60^{\circ} \mathrm{C}$ and $45 \mathrm{sec}$ at $72^{\circ} \mathrm{C}$. The threshold cycle $(\mathrm{Cq})$ was used to estimate the amount of target mRNA. The comparative $\mathrm{Cq}$ method with a formula for relative $\mathrm{FC}\left(2^{-\Delta \Delta \mathrm{Cq}}\right)$ was used to quantify the amplified transcripts $(32,33,41)$. The relative gene expression was determined via normalization to GAPDH. Experiments were evaluated in triplicate and repeated $\geq 3$ times.

Statistical analysis. Data are presented as the mean \pm SEM, and were analyzed using GraphPad Prism software v5.0 (GraphPad Software, Inc.). RT-qPCR parameters were analyzed using unpaired t-test for repeated measures. $\mathrm{P}<0.05$ was considered to indicate a statistically significant difference.

\section{Results}

Characteristics of ischemic myocardial tissues. Serum cTnI levels in the model group were significantly increased compared with the control group (Fig. 1B). In addition, the results of TTC staining (Fig. 1A), H\&E staining and Masson staining (Fig. 1C) all confirmed the successful establishment of the rat MIRI model. In the control group, the myocardium had a physiological myocardial architecture, and the myocardial fibres and myocardial cells were complete and arranged in an orderly manner. In the model group, structural disorder of the cardiac tissue was observed, with different degrees of vacuolar degeneration and necrosis, as well as loose stroma. Moreover, the number of cardiomyocyte fibres were markedly increased after MIRI.

mRNAs and lncRNAs expression profiles in MIRI. The patterns of the mRNA and lncRNA expressions in the T1-T4 spinal cord at $2 \mathrm{~h}$ after MIRI or sham operations were then examined using microarray. From the volcano map and hierarchical clustering analysis results, a landscape of the expression characteristics of the mRNAs and lncRNAs was obtained. The volcano plots demonstrated that large numbers of the mRNAs and IncRNAs were differentially expressed between the two groups (Fig. 2A and C). The hierarchical cluster analysis of the IncRNAs and mRNAs indicated that the samples of two groups were clustered together and the signal intensity was consistent in the two groups (Fig. 2B and D). Furthermore, these differential alterations of the mRNAs and lncRNAs in the T1-T4 spinal cord were associated with MIRI. 
Table I. Primer sequences for reverse transcription-quantitative PCR.

\begin{tabular}{|c|c|}
\hline \multicolumn{2}{|l|}{ A, mRNA } \\
\hline Primer name & Primer sequences $\left(5^{\prime} \rightarrow 3^{\prime}\right)$ \\
\hline \multirow[t]{2}{*}{ Frs3 } & F: GAGCCAGTCATCATCACACGGAAC \\
\hline & R: GAAGCCATTGGAGAAGCTGGAGAC \\
\hline \multirow[t]{2}{*}{ Zfp523 } & F: CGCTGACTTCCTGTGAGTGTGAC \\
\hline & R: ACCTTGGCTCTGGCTCTCGTC \\
\hline \multirow[t]{2}{*}{ Dnajc6 } & F: GACAAGCCTCATGGAGCCAAGAAG \\
\hline & R: GGAGAAGTTCGTGCTCACATCGG \\
\hline \multirow[t]{2}{*}{ Nedd41 } & F: TACGCAGTGGCACCGACCTAG \\
\hline & R: GTGTGCGGCCTCCTGATTGATC \\
\hline \multirow[t]{2}{*}{ Tep1 } & F: GGATGGATACGGAGCTGCTGAATG \\
\hline & R: GGACACTGACGAAGAGGCACTTG \\
\hline \multirow[t]{2}{*}{ Myef2 } & F: CCAGGTGGACAGCCAATTAGTGC \\
\hline & R: GCCTGCCATGCTATTCATTGCTTC \\
\hline \multirow[t]{2}{*}{ Tgfbr1 } & F: CATTGCTGGTCCAGTCTGCTTCG \\
\hline & R: TGGTGAATGACAGTGCGGTTATGG \\
\hline \multirow[t]{2}{*}{ Fgf12 } & F: TGCTGTGCGACTGTGAGATTGC \\
\hline & R: CGTGCGGCTCGTTGTACTCG \\
\hline \multirow[t]{2}{*}{ Mef2c } & F: TGCTGTGCGACTGTGAGATTGC \\
\hline & R: CGTGCGGCTCGTTGTACTCG \\
\hline \multirow[t]{2}{*}{ Tfdp1 } & F: AAGAAGATGCCGCCGAGTTGTG \\
\hline & R: CGCTGGCTTCACGACACATCC \\
\hline \multirow[t]{2}{*}{ GAPDH } & F: CGCTAACATCAAATGGGGTG \\
\hline & R: TTGCTGACAATCTTGAGGGAG \\
\hline
\end{tabular}

B, Long non-coding RNA

Primer sequences $\left(5^{\prime} \rightarrow 3^{\prime}\right)$

ENSRNOT00000080713

ENSRNOT00000090564

ENSRNOT00000082588

ENSRNOT00000091080

ENSRNOT00000091570

ENSRNOT00000087777

ENSRNOT00000082061

ENSRNOT00000091108

ENSRNOT00000087028

ENSRNOT00000086475
F: CACTTCCGCCCACATCACA

R: AAACCCCTCAGTCTCAAAACCT

F: GACCACGCATCTACA

R: GCGAACTGAGGTGAGAAGGGA

F: TGCTTCACTTCTCCCACTCTTC

R: AGGCCTTCCATCAAACAAATC

F: GGTGGTGGACAAGGATGAG

R: TGGACGGAGGTTGGGGAGAT

F: CAAGAAATCCAATACGACCC

R: TCCCCGTGGAAATAGGTGTAA

F: GGAAGAGGGACGTTGGGTA

R: AGCCAGGTCCAAGTCACAGG

F: CATTTCTTCCTCCCTTCCCTT

R: ACGGTTCTCCAATCGCACA

F: CCGTGGAATATGGGAAGGAC

R: CAAGATGGAAAGGCAACGAG

F: ACGGGGTGAAAGGGAAGA

R: TGCTGGTTTGTTAAGGGGATG

F: CAGTTTGGTGTCTGTTTCCC

R: TGCTTCTTCGAGCCGGTATT

F, forward; R, reverse. 

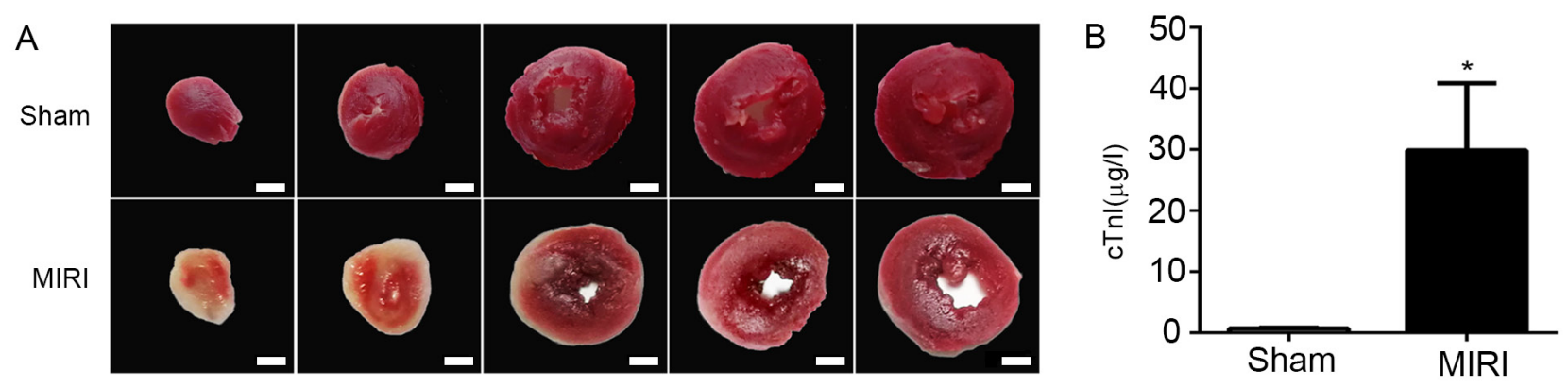

C

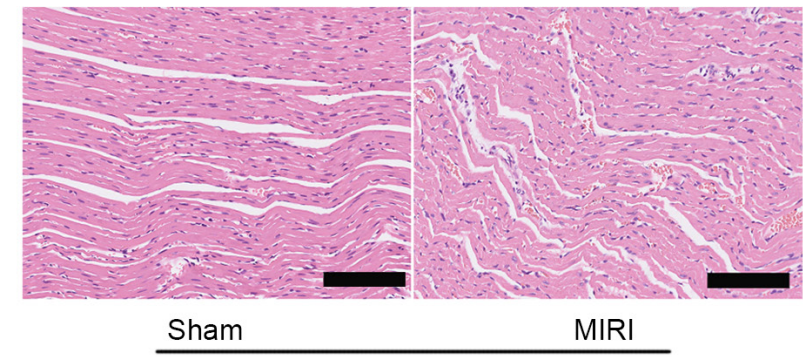

H\&E

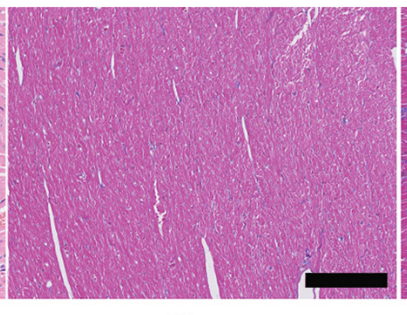

Sham

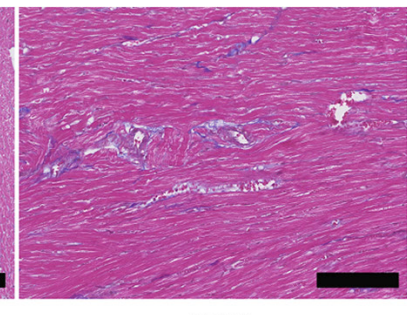

MIRI

Masson

Figure 1. Evaluation of MIRI. (A) Representative images of 2,3,5-triphenyltetrazolium chloride staining (left) and quantitative analysis of infarct size (right) in hearts subjected to Sham and MIRI surgery. Scale bar, $2 \mathrm{~mm}$. (B) Serum cTnI concentrations in rats with Sham or MIRI surgery. Data are presented as the mean \pm SEM, $n=4$ rats per group. (C) Representative images of H\&E staining and Masson trichrome staining from rat left ventricles $2 \mathrm{~h}$ after MIRI and in sham group. Scale bar, $100 \mu \mathrm{m}$. ${ }^{*} \mathrm{P}<0.05$ vs. sham group. H\&E, hematoxylin and eosin; MIRI, myocardial ischemia-reperfusion injury.

Differentially expressed mRNAs and IncRNAs. To further analyze the differentially expressed mRNAs and lncRNAs, a significance analysis of the microarrays method with the criteria $\mathrm{P}<0.05$ and $\mathrm{FC}>1$ was performed.

In the differentially expressed mRNAs, there were 470 genes that exhibited an FC that was $>1$. The number of downregulated mRNAs was 239, whereas the number of upregulated mRNAs was 231. The most upregulated mRNAs were Tfdp1, Map2, Cep19, Aurkaip1, Tpbg, Myef2, Ap2m1, Tgfbr1, Fgf12 and Mef2c. The most downregulated mRNAs were Frs3, Zfp523, Tpm4, Apold1, Ctnnb1, Lss, Psen2, Dnajc6, Nedd41 and Tep1. Detailed information regarding the differentially expressed mRNAs is listed in Table II.

The results identified that 126 lncRNAs, which included 41 upregulated and 85 downregulated IncRNAs, were significantly altered in the MIRI group compared with the sham group. The most upregulated lncRNAs were ENSRNOT00000086475, ENSRNOT00000087028, ENSRNOT00000091108, ENSRNOT00000031815, ENSRNOT00000082061 and ENSRNOT00000087777. The mostdownregulatedlncRNAs wereENSRNOT00000080713, ENSRNOT00000090564, ENSRNOT00000082588, ENSRNOT00000091080, ENSRNOT00000091570, ENSRNOT00000077319, ENSRNOT00000077509, ENSRNOT00000089288, ENSRNOT00000083187 and ENSRNOT00000091435. Additional information regarding the differentially expressed lncRNAs is presented in Table III.

Functional prediction of differentially expressed $m R N A s$ in MIRI. To investigate the spinal molecular mechanisms in MIRI, GO and KEGG pathway analyses of the differentially expressed mRNAs in MIRI vs. sham were performed.
To identify major biochemical metabolic pathways and signal transduction pathways for differential gene enrichment, KEGG pathway analysis (Fig. 3) was conducted. Using the KEGG pathway analysis, it was found that differential genes were mainly enriched in the 'PI3K/Akt signaling pathway', 'protein digestion and uptake', 'p53 signaling pathway' and 'neuroactive ligand-dependent body interaction' (Fig. 3; Table IV).

The differential genes associated with cellular components were mainly enriched in the 'extracellular fraction', 'cytoplasm', 'microtubule cytoskeleton', 'cytoskeleton' and 'intracellular', whereas the genes participating in biological processes were mainly enriched in 'cell signal transduction' (Fig. 4; Table V).

Gene co-expression networks were presented to identify interactions between IncRNAs and their co-expression genes (Fig. 5). The co-expressed network showed that 10 highly-dysregulated lncRNAs were highly co-expressed with 277 target genes, of which 10 were positively correlated (colored with yellow) and 267 were negatively correlated (colored with blue). ENSRNOT00000080713 was at the core of the lncRNA-mRNA co-expression network and had a co-expression relationship with many mRNAs.

$R T-q P C R$ confirmation of $m R N A$ and IncRNA expression from microarray. To confirm the reliability of the microarray data and analyze the temporal alterations in mRNA and lncRNA expression after MIRI, the IncRNAs, ENSRNOT00000080713, ENSRNOT00000090564, ENSRNOT00000082588, ENSRNOT00000091080, ENSRNOT00000091570, ENSRNOT00000087777, ENSRNOT00000082061, ENSRNOT00000091108, ENSRNOT00000087028 and ENSRNOT00000086475, and the mRNAs, fibroblast growth factor receptor substrate 3 (Frs3), zinc finger protein 76 
A

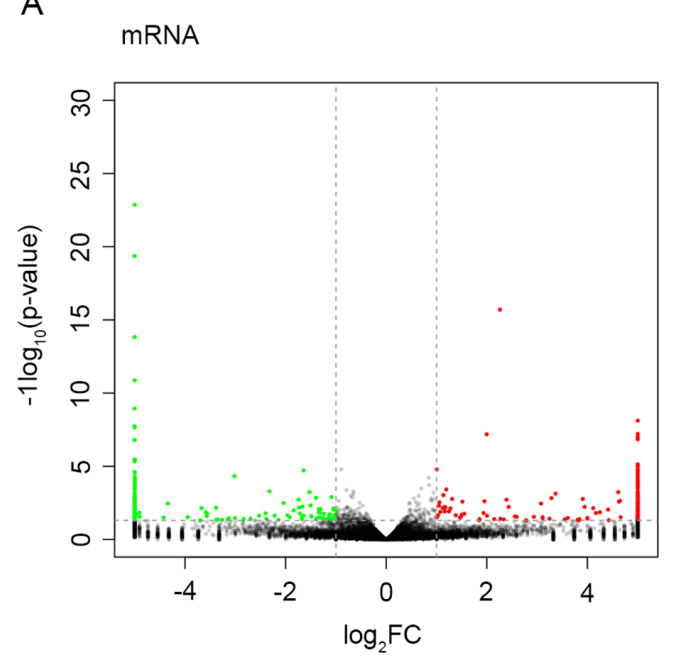

C

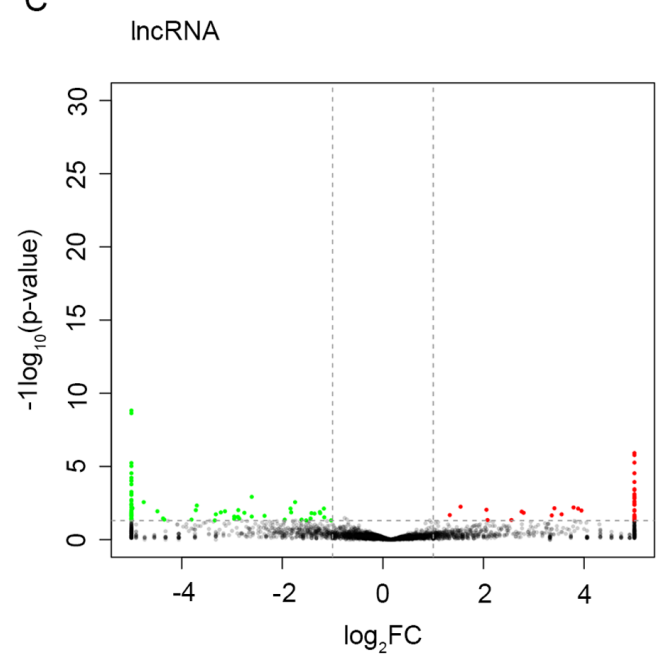

B
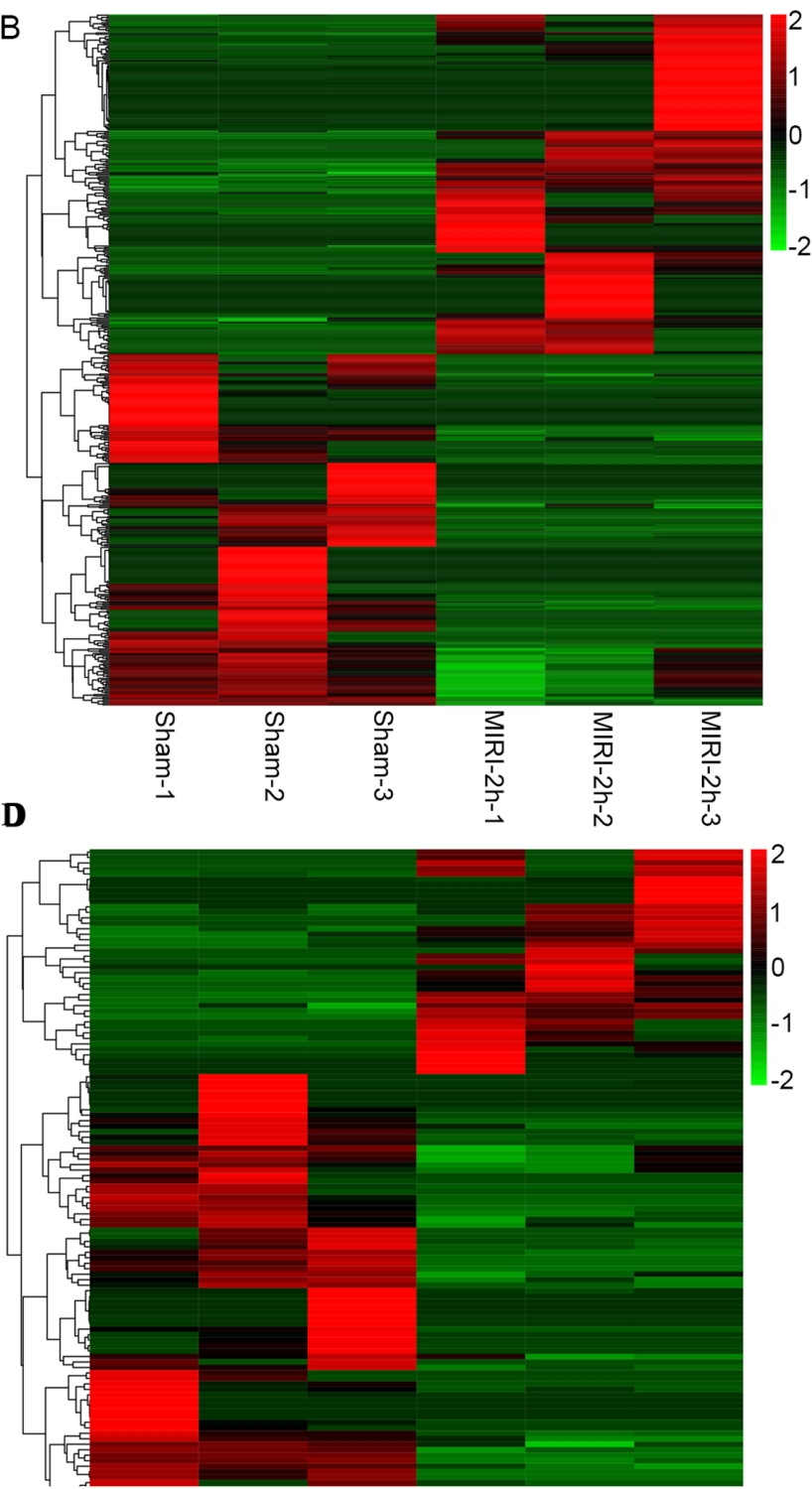

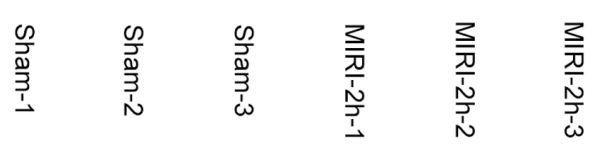

Figure 2. MIRI results in expression profile changes of mRNA and lncRNA in the T1-T4 spinal cord. (A) Volcano plot comparing global mRNA gene expression profiles in the spinal cord between the two groups. (B) Hierarchical cluster analysis of the mRNAs. (C) Volcano plot comparing global lncRNA gene expression profiles in the spinal cord between the two groups. (D) Hierarchical cluster analysis of the lncRNAs. The cluster analysis demonstrated that the three MIRI or three sham samples were clustered together. Upregulated, downregulated and not statistically different genes were colored in red, green and black, respectively. MIRI, myocardial ischemia-reperfusion injury; lncRNA, long non-coding RNA.

(Zfp523), DnaJ heat shock protein family (Hsp40) member C6 (Dnajc6), NEDD4 like E3 ubiquitin protein ligase (Nedd4l), telomerase associated protein 1 (Tep1), myelin expression factor 2 (Myef2), transforming growth factor $\beta$ receptor 1 (Tgfbr1), fibroblast growth factor 12 (Fgf12), myocyte enhancer factor 2C (Mef2c) and transcription factor Dp-1 (Tfdp1), were randomly selected and detected via RT-qPCR.

The results demonstrated that expression of lncRNAs ENSRNOT00000080713, ENSR NOT00000090564, ENSRNOT00000082588, ENSRNOT00000091080 and ENSRNOT00000091570 were markedly decreased in MIRI group compared with those in the control group, whilst ENSRNOT00000087777, ENSRNOT00000082061,
ENSRNOT00000091108, ENSRNOT00000087028 and ENSRNOT00000086475 were markedly increased. In addition, the mRNAs Frs3, Zfp523, Dnajc6, Nedd41 and Tep1 were markedly decreased in the MIRI group compared with those in the control group, but Myef2, Tgfbr1, Fgf12, Mef2c and Tfdp1 were markedly increased. These RT-qPCR results were consistent with the results from the transcriptome sequencing (Fig. 6), which further supported the reliability of the microarray data.

$R T$-qPCR validation of mRNAs and IncRNAs in T1-T4 spinal cord. The results of RT-qPCR demonstrated that the mRNA expression levels of Frs3, Nedd41 and Tep1 were 
Table II. Details of the top 10 downregulated and upregulated mRNAs in the T1-T4 spinal cord between MIRI and sham groups.

A, Downregulation

\begin{tabular}{|c|c|c|c|c|}
\hline ID & $\begin{array}{c}\log _{2} \mathrm{FC} \\
(\mathrm{MIRI} / \text { sham })\end{array}$ & Symbol & P-value & Description \\
\hline ENSRNOT00000019404 & -12.0846 & Frs3 & $4.50 \times 10^{-20}$ & Fibroblast growth factor receptor substrate 3 \\
\hline ENSRNOT00000000595 & -11.2761 & Zfp523 & $1.51 \times 10^{-14}$ & Zinc finger protein 523 \\
\hline ENSRNOT00000089056 & -10.8893 & Tpm4 & 0.001717 & Tropomyosin 4 \\
\hline ENSRNOT00000010289 & -10.5635 & Apold1 & $1.1610^{-9}$ & Apolipoprotein L domain containing 1 \\
\hline ENSRNOT00000079085 & -10.1716 & Ctnnb1 & $8.32 \times 10^{-5}$ & Catenin beta 1 \\
\hline ENSRNOT00000081903 & -9.81911 & Lss & 0.013918 & $\begin{array}{l}\text { Lanosterol synthase (2,3-oxido squalene-lanos terol } \\
\text { cyclase) }\end{array}$ \\
\hline ENSRNOT00000091715 & -9.75933 & Psen2 & 0.000372 & Presenilin 2 \\
\hline ENSRNOT00000086539 & -9.30682 & Dnajc6 & $5.25 \times 10^{-5}$ & DnaJ heat shock protein family (Hsp40) member C6 \\
\hline ENSRNOT00000083363 & -9.15482 & Nedd41 & 0.005334 & $\begin{array}{l}\text { Neural precursor cell expressed, developmentally } \\
\text { down-regulated 4-like, E3 ubiquitin protein ligase }\end{array}$ \\
\hline ENSRNOT00000088981 & -8.70275 & Tep1 & 0.001598 & Telomerase associated protein 1 \\
\hline
\end{tabular}

B, Upregulation

\begin{tabular}{|c|c|c|c|c|}
\hline ID & $\begin{array}{c}\log _{2} \mathrm{FC} \\
\text { (MIRI/sham) }\end{array}$ & Symbol & P-value & Description \\
\hline ENSRNOT00000087192 & 12.22179 & Tfdp1 & $6.42 \times 10^{-8}$ & Transcription factor Dp-1 \\
\hline ENSRNOT00000045766 & 11.86754 & Map2 & $5.07 \times 10^{-5}$ & Microtubule-associated protein 2 \\
\hline ENSRNOT00000037795 & 11.22882 & Cep19 & $2.22 \times 10^{-5}$ & Centrosomal protein 19 \\
\hline ENSRNOT00000025531 & 11.16113 & Aurkaip1 & 0.000724 & Aurora kinase A interacting protein 1 \\
\hline ENSRNOT00000014326 & 10.76763 & Tpbg & 0.000962 & Trophoblast glycoprotein \\
\hline ENSRNOT00000081533 & 10.69407 & Myef2 & $7.98 \times 10^{-9}$ & Myelin expression factor 2 \\
\hline ENSRNOT00000088821 & 10.64386 & Ap2m1 & 0.004070 & Adaptor-related protein complex 2 , mu 1 subunit \\
\hline ENSRNOT00000081090 & 9.813781 & Tgfbr1 & 0.005577 & Transforming growth factor, beta receptor 1 \\
\hline ENSRNOT00000071586 & 9.781360 & Fgf12 & 0.004286 & Fibroblast growth factor 12 \\
\hline ENSRNOT00000076230 & 8.748193 & Mef2c & $1.98 \times 10^{-5}$ & Myocyte enhancer factor $2 \mathrm{C}$ \\
\hline
\end{tabular}

FC, fold change; T, thoracic; MIRI, Myocardial ischemia-reperfusion injury.

significantly downregulated in the model group, while the expression of mRNA Myef2 was significantly upregulated in the model group. The expression levels of mRNA Zfp523, Dnajc6 and Myef2 were not significantly different between the two groups (Fig. 7). The expression levels of lncRNA ENSRNOT00000080713, ENSRNOT00000090564, ENSRNOT00000082588 and ENSRNOT00000091570 were significantly downregulated in the model group, but the expression levels of lncRNA ENSRNOT00000087777, ENSRNOT00000082061, ENSRNOT00000087028 and ENSRNOT00000086475 were significantly upregulated in the model group. However, the expression levels of lncRNA ENSRNOT00000091080 and ENSRNOT00000091108 were not significantly different between the two groups (Fig. 8).

\section{Discussion}

MIRI causes nociceptive chemical and electrical signals that affect dorsal root ganglia and the upper thoracic spinal cord (42).
Previous studies have reported that spinal gene expression is associated with in the development of MIRI $(9,27)$ and have identified the molecular mechanisms during MIRI $(26,27,42)$; however, the impact of the spinal process during MIRI requires further investigation.

Emerging evidence indicates that the inflammatory and immune responses that occur in the spinal cord are vital contributors to the progression of MIRI $(9,13,14,16)$. Moreover, autonomic dysregulation following MIRI is an important pathogenic event (43-47). Interactions between the heart and spinal autonomic nervous system serve a role in both the development and maintenance of MIRI, and are also crucial in myocardial ischemia-induced angina pectoris (48-52). Based on the importance of the spinal autonomic nervous system in the pathophysiology of MIRI, there has been a notable effort in this field to study changes in the genomics, transcriptomics, proteomics and metabolomics in the spinal cord after MIRI, which could facilitate both existing interventions and novel therapies directed at cardiac-autonomic targets (53). 
Table III. Details of the downregulated and upregulated long non-coding RNAs in the T1-T4 spinal cord between MIRI and sham group.

A, Downregulation

\begin{tabular}{llll}
\hline ID & Log2FC (MIRI/sham) & P-value & Symbol \\
\hline ENSRNOT00000080713 & -9.98726 & 0.000442 & LOC102550026 \\
ENSRNOT00000090564 & -8.10329 & 0.040576 & AABR07050135.1 \\
ENSRNOT00000082588 & -7.49185 & $4.01 \times 10^{-5}$ & AABR07000385.2 \\
ENSRNOT00000091080 & -6.9542 & $1.14 \times 10^{-6}$ & AABR07044980.1 \\
ENSRNOT00000091570 & -6.71425 & 0.025341 & AABR07071198.1 \\
ENSRNOT00000077319 & -6.22882 & 0.005841 & AABR07071383.1 \\
ENSRNOT00000077509 & -5.32193 & 0.048185 & AABR07027371.1 \\
ENSRNOT00000089288 & -4.16993 & 0.01174 & AABR07044001.4 \\
ENSRNOT00000083187 & -3.52356 & 0.024012 & AABR07060560.4 \\
ENSRNOT00000091435 & -3.38082 & 0.016282 & AABR07042668.1 \\
\hline
\end{tabular}

B, Upregulation

\begin{tabular}{lccc}
\hline ID & Log2FC (MIRI/sham) & P-value & Symbol \\
\hline ENSRNOT00000086475 & 8.797662 & 0.013366 & AABR07070801.2 \\
ENSRNOT00000087028 & 6.129283 & 0.027308 & LOC103692514 \\
ENSRNOT00000091108 & 4.058894 & 0.020796 & AABR07006724.1 \\
ENSRNOT00000031815 & 3.297681 & 0.041965 & Rn50_X_0744.6 \\
ENSRNOT00000082061 & 2.966833 & 0.034871 & AABR07071395.1 \\
ENSRNOT00000087777 & 2.917538 & 0.024331 & AABR07016845.1 \\
\hline
\end{tabular}

FC, fold change; T, thoracic; MIRI, Myocardial ischemia-reperfusion injury.

Table IV. Differential gene enriched in Kyoto Encyclopedia of Genes and Genomes pathway.

\begin{tabular}{llrr}
\hline Pathway ID & \multicolumn{1}{c}{ Pathway } & Gene number & P-value \\
\hline ko04151 & PI3K/Akt signaling pathway & 20 & 0.00039 \\
ko04974 & Protein digestion and absorption & 8 & 0.001448 \\
ko04115 & p53 signaling pathway & 7 & 0.001795 \\
ko04510 & Focal adhesion & 13 & 0.005058 \\
ko04080 & Neuroactive ligand-receptor interaction & 16 & 0.005635 \\
ko04512 & ECM-receptor interaction & 7 & 0.008425 \\
ko00534 & Glycosaminoglycan biosynthesis-heparan sulfate/heparin & 3 & 0.019966 \\
ko04960 & Aldosterone-regulated sodium reabsorption & 4 & 0.023467 \\
ko04015 & Rap1 signaling pathway & 5 & 0.027612 \\
ko04721 & Synaptic vesicle cycle & 4 & 0.030579 \\
ko00600 & Sphingolipid metabolism & 11 & 0.036733 \\
ko04020 & Calcium signaling pathway & 0.03857
\end{tabular}

ECM, extracellular matrix.

In the present study, the differentially expressed lncRNAs in the T1-T4 spinal cord during MIRI, along with their characteristics and possible relationships with mRNAs, were identified. A total of 126 lncRNAs were observed in the T1-T4 spinal cord of the MIRI model rats. Among these lncRNAs,
41 lncRNAs were upregulated, and 85 lncRNAs were downregulated at $2 \mathrm{~h}$ after MIRI. Moreover, RT-qPCR was used to validate the microarray analysis results, for which the conclusion was consistent. Based on these findings, it was suggested that the aforementioned lncRNAs may serve a role in MIRI 
Table V. Functional classification of differential genes via GO analysis.

A, Cellular component

\begin{tabular}{llrr}
\hline GO ID & \multicolumn{1}{c}{ Description } & Gene number & P-value \\
\hline GO:0044424 & Intracellular part & 48 & 0.013126 \\
GO:0005737 & Cytoplasm & 30 & 0.025862 \\
GO:0015630 & Microtubule cytoskeleton & 3 & 0.026874 \\
GO:0005856 & Cytoskeleton & 7 & 0.041896 \\
GO:0005622 & Intracellular & 63 & 0.044992 \\
\hline
\end{tabular}

B, Biological process

\begin{tabular}{llll}
\hline GO ID & \multicolumn{1}{c}{ Description } & Gene number & P-value \\
\hline GO:0030198 & Extracellular matrix organization & 3 & 0.010728 \\
GO:0043062 & Extracellular structure organization & 3 & 0.010729 \\
GO:0007267 & Cell-cell signaling & 4 & 0.028507 \\
GO:0032196 & Transposition & 1 & 0.035381 \\
\hline
\end{tabular}

GO, Gene Ontology.

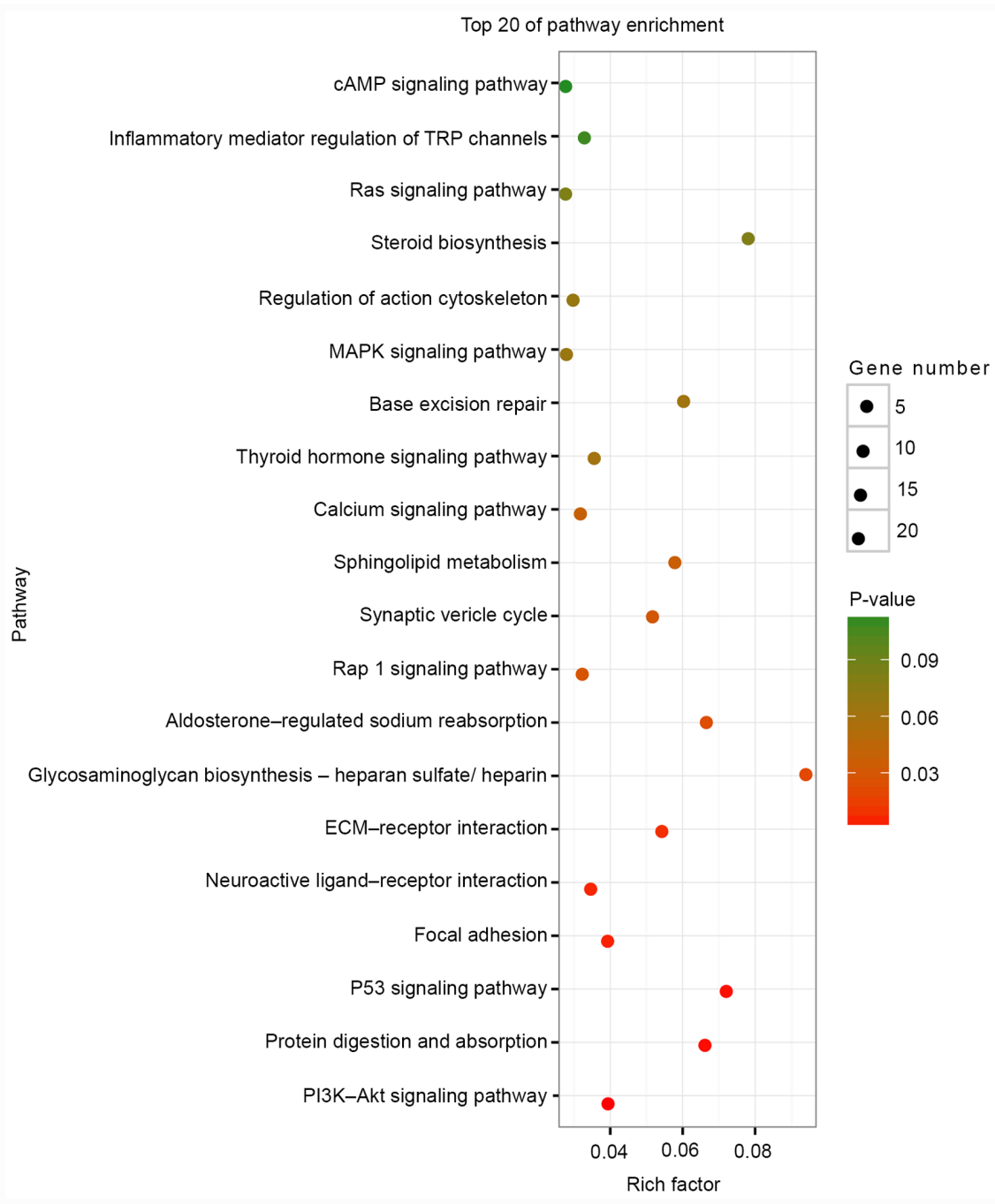

Figure 3. Heatmap of Kyoto Encyclopedia of Genes and Genomes pathway analysis. Size of the bubble represents the number of enriched genes, and the color of the bubble from red to green indicates a gradual increase in the P-value. ECM, extracellular matrix; TRP, transient receptor potential. 


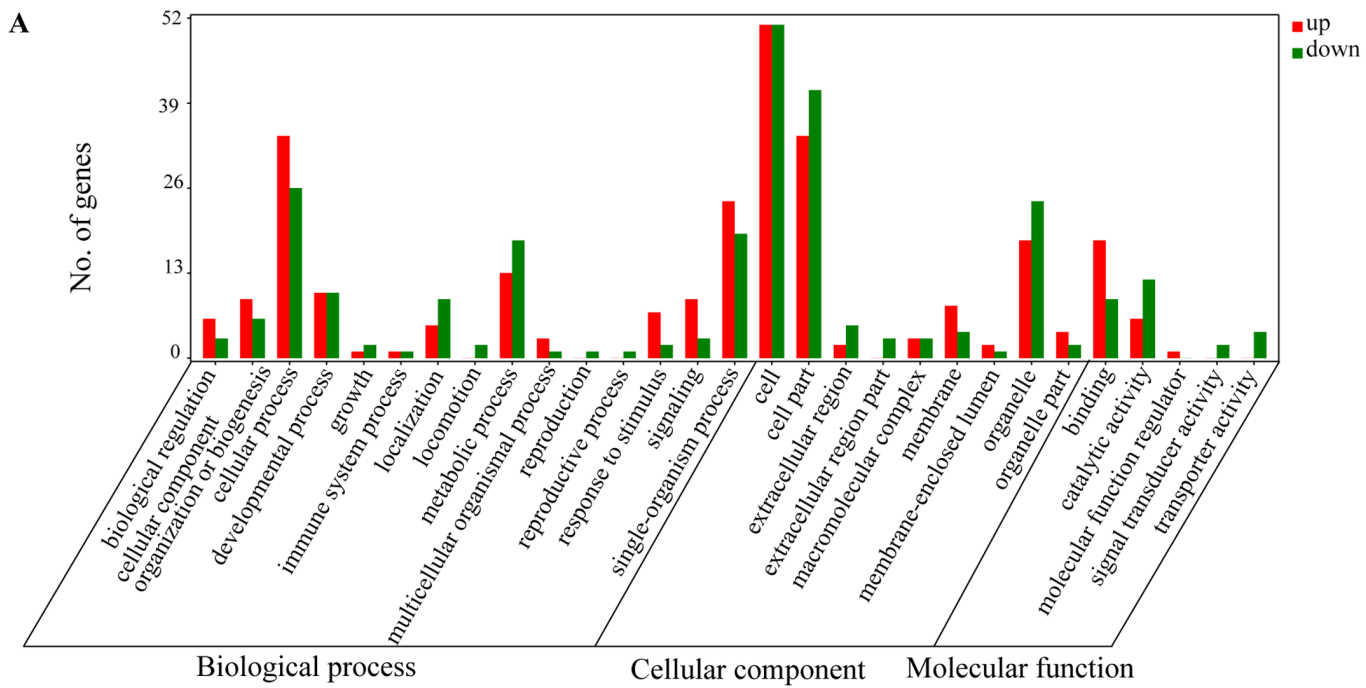

B

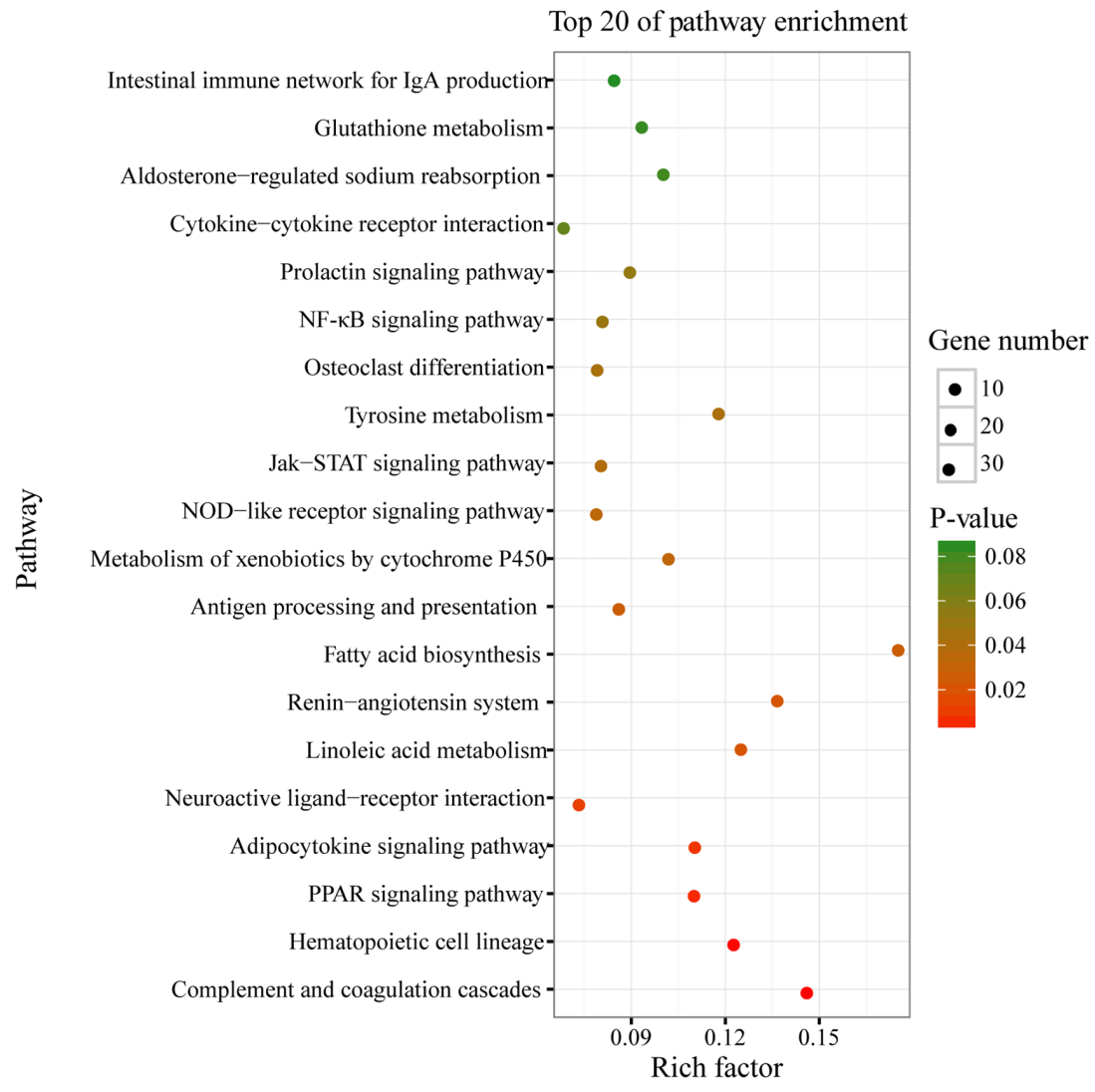

Figure 4. Functional analysis of differential lncRNAs. (A) Gene Ontology analysis heat map of co-expressed genes of differential lncRNAs. (B) Kyoto Encyclopedia of Genes and Genomes pathway analysis heat map of co-expressed genes of differential lncRNAs. IncRNA, long non-coding RNA; PPAR, peroxisome proliferator activated receptor; NOD, nucleotide-binding oligomerization domain-containing protein.

pathology. Furthermore, the present research contributes to understanding the molecular mechanism of MIRI.

To further investigate the roles of these differentially expressed lncRNAs in the development of MIRI, GO and KEGG pathway analyses were performed. Based on the GO and KEGG enrichment analyses of these lncRNAs, the results suggested that the significantly enriched biologic processes and molecular functions of the upregulated genes after MIRI were associated with gene sets termed as follows: 'PI3K/Akt signaling pathway', 'protein digestion and absorption', 'p53 signaling pathway' and 'neuroactive ligand-dependent body interaction'.
The PI3K/Akt signaling pathway is associated with MIRI (54). p53 is a tumor suppressor, which exerts an important role in the process of apoptosis and is associated with the occurrence and development of cardiovascular disease (55-57). Previous studies have shown that apoptosis is a typical manifestation and primary pathological mechanism of MIRI (58). The loss of cardiomyocytes caused by apoptosis is an important reason for the development of various heart diseases (59). Preventing the process of apoptosis can prevent myocardial damage caused by reperfusion, thus slowing or preventing the occurrence of heart failure and even mortality (60). 


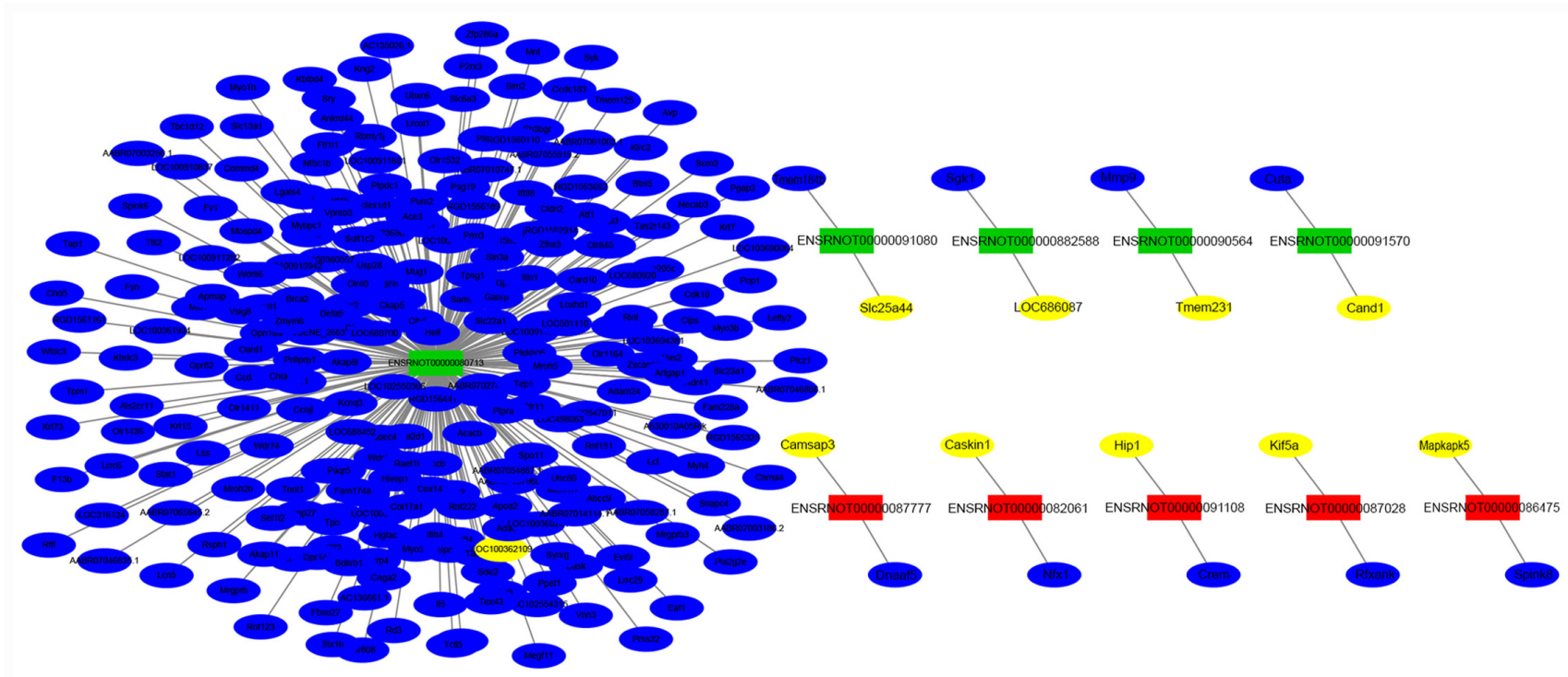

Figure 5. Sub-network of 10 highly dysregulated lncRNAs co-expressed with mRNAs. Genes colored in red are upregulated lncRNAs and those in green are downregulated lncRNAs. Upregulated mRNAs are colored with yellow and downregulated mRNAs are colored with blue. IncRNA, long non-coding RNA.
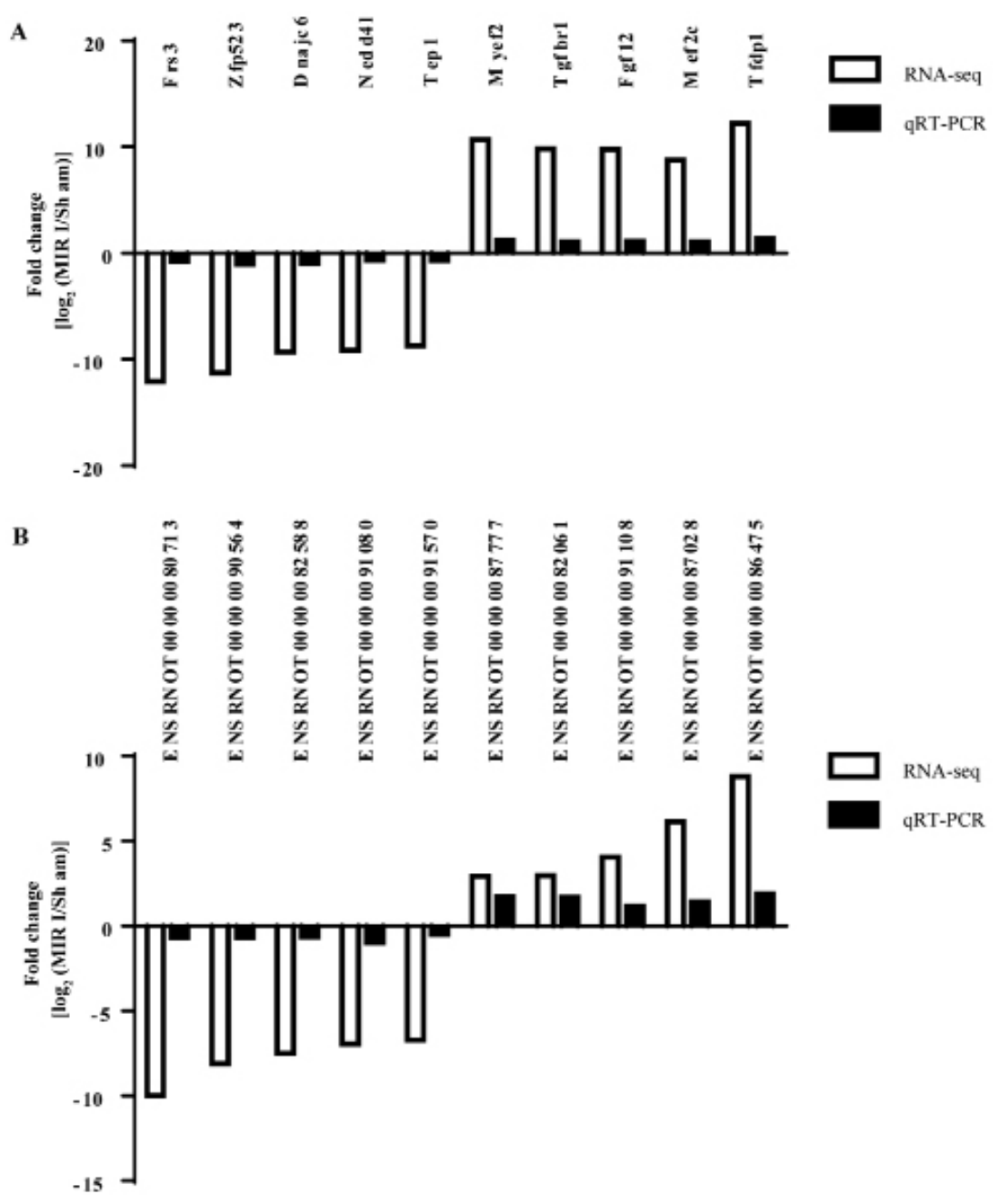

Figure 6. RT-qPCR confirmation of mRNA and lncRNA expression from the microarray. (A) mRNA expression levels in spinal cord tissue of MIRI rats. (B) lncRNA expression levels in spinal cord tissue of MIRI rats. The expression of mRNAs and lncRNAs in the spinal cord of rats with MIRI was consistent with the results of transcriptome sequencing, indicating the reliability of the microarray data. IncRNA, long non-coding RNA; Frs3, fibroblast growth factor receptor substrate 3; Zfp523, zinc finger protein 76; Dnajc6, DnaJ heat shock protein family (Hsp40) member C6; Nedd4l, NEDD4 like E3 ubiquitin protein ligase; Tep1, telomerase associated protein 1; Myef2, myelin expression factor 2; Tgfbr1, transforming growth factor $\beta$ receptor 1; Fgf12, fibroblast growth factor 12; Mef2c, myocyte enhancer factor 2C; Tfdp1, transcription factor Dp-1; RT-qPCR, reverse transcription-quantitative PCR; seq, sequencing; MIRI, myocardial ischemia-reperfusion injury. 

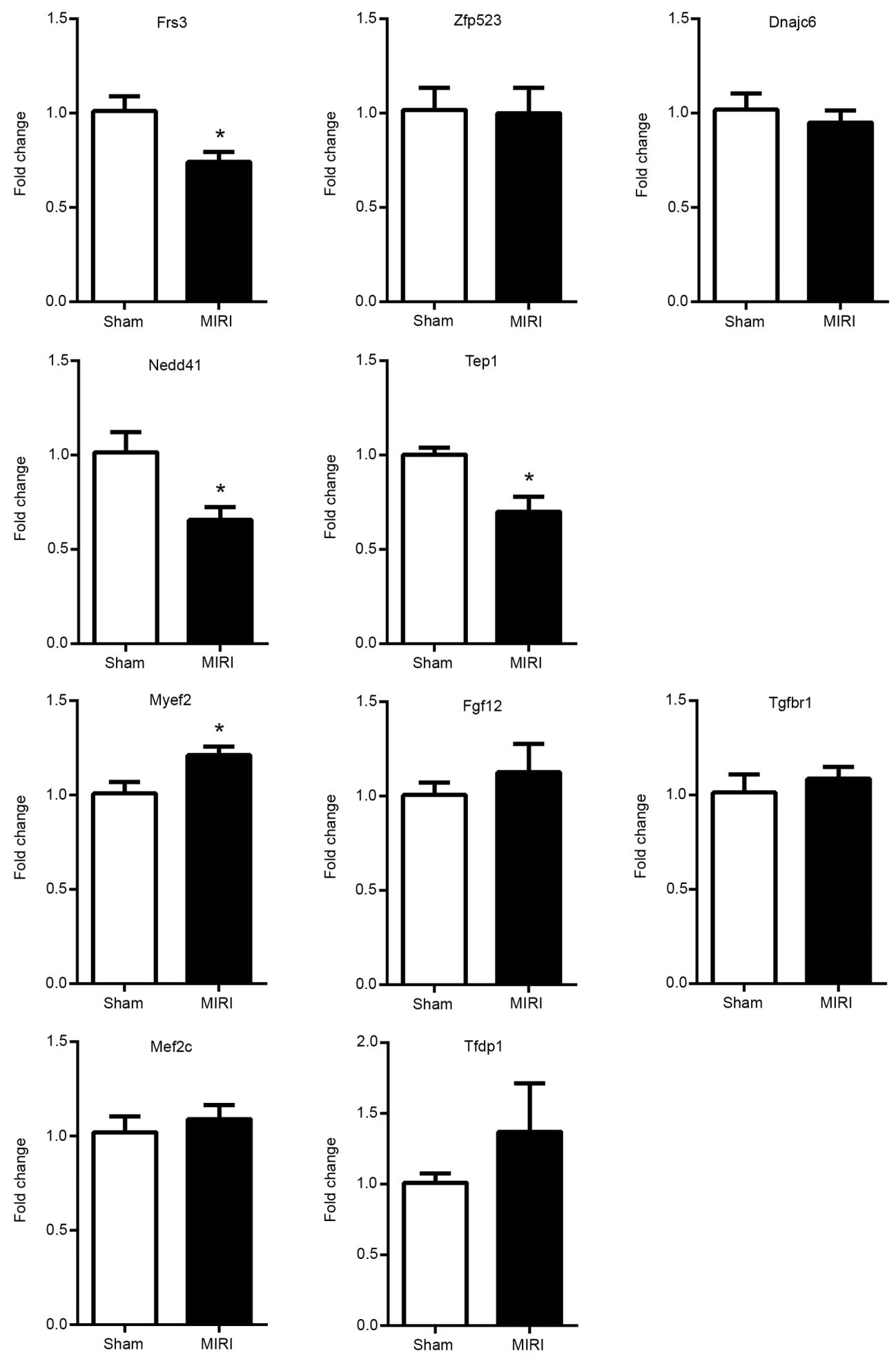

Figure 7. Reverse transcription-quantitative PCR validation of the mRNAs in the T1-T4 spinal cord. The expression levels of mRNA Frs3, Nedd41 and Tep1 were significantly downregulated in model group, whereas the expression of mRNA Myef2 was significantly upregulated. The expression levels of mRNA Zfp523, Dnajc6 and Myef2 were not statistically different between the two groups. Data are presented as the mean \pm SEM $(n=6)$. " $P<0.05$ vs. control. Frs3, fibroblast growth factor receptor substrate 3; Zfp523, zinc finger protein 76; Dnajc6, DnaJ heat shock protein family (Hsp40) member C6; Nedd4l, NEDD4 like E3 ubiquitin protein ligase; Tep1, telomerase associated protein 1; Myef2, myelin expression factor 2; Tgfbr1, transforming growth factor $\beta$ receptor 1; Fgf12, fibroblast growth factor 12; Mef2c, myocyte enhancer factor 2C; Tfdp1, transcription factor Dp-1; MIRI, myocardial ischemia-reperfusion injury.

The present study has several limitations. For example, the reperfusion time for tissue collection lasted only $2 \mathrm{~h}$. Therefore, it remains unknown if the expression of lncRNA has the same regulation for a longer period after ischemia. Moreover, a functional analysis of differentially expressed genes was performed without further verification. LncRNAs are poorly conserved and only part of the sequence is conserved by multiple species $(61,62)$. Previous studies have reported that there are 481 segments longer $>200 \mathrm{bp}$ (range, 200-779 bp) that are conserved between orthologous regions of the mouse, rat 

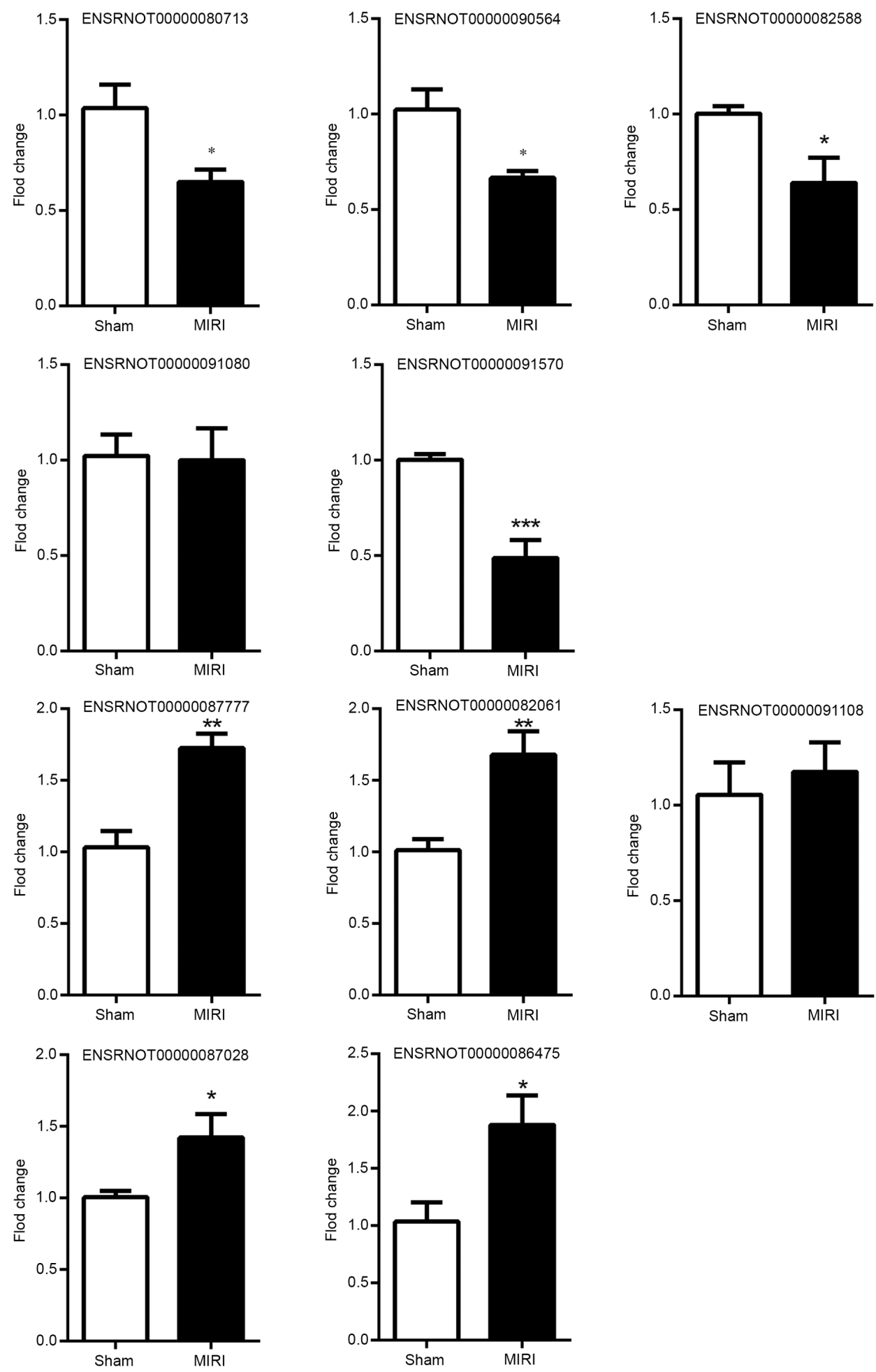

Figure 8. Reverse transcription-quantitative PCR validation of 10 lncRNAs in the T1-T4 spinal cord. The expression levels of lncRNA ENSRNOT00000080713, ENSRNOT00000090564, ENSRNOT00000082588 and ENSRNOT00000091570 were significantly downregulated in model group, whereas the expression levels of IncRNA ENSRNOT00000087777, ENSRNOT00000082061, ENSRNOT00000087028 and ENSRNOT00000086475 were significantly upregulated. The expression levels of IncRNA ENSRNOT00000091080 and ENSRNOT00000091108 were not statistically different between the two groups. Data are presented as the mean $\pm \mathrm{SEM}(\mathrm{n}=6) .{ }^{*} \mathrm{P}<0.05,{ }^{* *} \mathrm{P}<0.01,{ }^{* * *} \mathrm{P}<0.001$ vs. control. MIRI, myocardial ischemia-reperfusion injury; lncRNA, long non-coding RNA.

and human genomes (63), and these conserved lncRNAs can provide an insight into their key functional roles $(64,65)$. As a result, further research is required to examine the functions of lncRNAs and the conservation of differentially expressed IncRNAs in rats and humans, which will identify their predicted targets and provide a detailed expression profile of lncRNA.
In conclusion, the present study demonstrated that lncRNA transcripts of the upper thoracic spinal cord were differentially expressed after MIRI. These spinal lncRNAs may regulate the expression of MIRI-related proteins and genes, and contribute to the pathogenesis of MIRI. Future investigations of these spinal lncRNAs found in the present study will mainly focus on 
their functions and their relationship with MIRI. Nonetheless, the present study may provide important evidence for further basic research and clinical investigations on improved therapeutic interventions of MIRI.

\section{Acknowledgements}

Not applicable.

\section{Funding}

This work was supported by grants from National Natural Science Foundation of P.R. China (grant nos. 82070302, 81873467 and 81770283), the Clinical Medical Research Center of Peritoneal Cancer of Wuhan (grant no. 2015060911020462), Research Foundation of Health and Family Planning Commission of Hubei Province (grant nos. WJ2015MA010 and WJ2017M249), Natural Science Foundation of Hubei Province (grant no. 2015CFA027) and the National Natural Science Foundation of Hubei Province (grant no. 2016CFB625).

\section{Availability of data and materials}

The datasets used and/or analyzed during the current study are available from the corresponding author on reasonable request.

\section{Authors' contributions}

ZXL and YJL performed the surgical procedures and PCR experiments. QW and ZGH participated in the experimental design. MHF and ZGH analyzed the data. HBX and MHF contributed to the study concept and design and supervised the whole project. All authors contributed to the final manuscript. All authors read and approved the final manuscript.

\section{Ethics approval and consent to participate}

The present study was approved by the Institutional Ethical Committee of Tongji Hospital, Tongji Medical College, Huazhong University of Science and Technology (approval no. TJ-A20150804).

\section{Patient consent for publication}

Not applicable.

\section{Competing interests}

The authors declare that they have no competing interests.

\section{References}

1. Khan MA, Hashim MJ, Mustafa H, Baniyas MY, Al Suwaidi SK, AlKatheeri R, Alblooshi FM, Almatrooshi ME, Alzaabi ME, Al Darmaki RS and Lootah SN: Global epidemiology of ischemic heart disease: Results from the global burden of disease study. Cureus 12: e9349, 2020.

2. Roth GA, Johnson C, Abajobir A, Abd-Allah F, Abera SF, Abyu G, Ahmed M, Aksut B, Alam T, Alam K, et al: Global, regional, and national burden of cardiovascular diseases for 10 causes, 1990 to 2015. J Am Coll Cardiol 70: 1-25, 2017.
3. Yellon DM and Hausenloy DJ: Myocardial reperfusion injury. N Engl J Med 357: 1121-1135, 2007.

4. Grace PA: Ischemia-reperfusion injury. Br J Surg 81: 637-647, 1994

5. Cheng YF, Chang YT, Chen WH, Shih HC, Chen YH, Shyu BC and Chen CC: Cardioprotection induced in a mouse model of neuropathic pain via anterior nucleus of paraventricular thalamus. Nat Commun 8: 826, 2017.

6. Xu A, Song Z, Peng Y and Xiang H: Change of mitochondrial function in the early stage after cardiac ischemia-reperfusion injury in mice. Int J Clin Exp Med 9: 2549-2554, 2016.

7. De Hert S and Moerman A: Myocardial injury and protection related to cardiopulmonary bypass. Best Pract Res Clin Anaesthesiol 29: 137-149, 2015.

8. Murphy E and Steenbergen C: Mechanisms underlying acute protection from cardiac ischemia-reperfusion injury. Physiol Rev 88: 581-609, 2008.

9. Saddic LA, Howard-Quijano K, Kipke J, Kubo Y, Dale EA, Hoover D, Shivkumar K, Eghbali M and Mahajan A: Progression of myocardial ischemia leads to unique changes in immediate-early gene expression in the spinal cord dorsal horn. Am J Physiol Heart Circ Physiol 315: H1592-H1601, 2018.

10. Waldron NH, Fudim M, Mathew JP and Piccini JP: Neuromodulation for the treatment of heart rhythm disorders. JACC Basic Transl Sci 4: 546-562, 2019.

11. Shen MJ and Zipes DP: Role of the autonomic nervous system in modulating cardiac arrhythmias. Circ Res 114: 1004-1021, 2014.

12. Hua F, Ardell JL and Williams CA: Left vagal stimulation induces dynorphin release and suppresses substance $P$ release from the rat thoracic spinal cord during cardiac ischemia. Am J Physiol Regul Integr Comp Physiol 287: R1468-R1477, 2004.

13. Steagall RJ, Sipe AL, Williams CA, Joyner WL and Singh K: Substance $\mathrm{p}$ release in response to cardiac ischemia from rat thoracic spinal dorsal horn is mediated by trpvl. Neuroscience 214: 106-119, 2012.

14. Ding X, Ardell JL, Hua F, McAuley RJ, Sutherly K, Daniel JJ and Williams CA: Modulation of cardiac ischemia-sensitive afferent neuron signaling by preemptive $\mathrm{C} 2$ spinal cord stimulation: Effect on substance P release from rat spinal cord. Am J Physiol Regul Integr Comp Physiol 294: R93-R101, 2008.

15. Gao C, Howard-Quijano K, Rau C, Takamiya T, Song Y, Shivkumar K, Wang Y and Mahajan A: Inflammatory and apoptotic remodeling in autonomic nervous system following myocardial infarction. PLoS One 12: e0177750, 2017.

16. Niu YL, Guo Z and Zhou RH: Up-Regulation of TNF-alpha in neurons of dorsal root ganglia and spinal cord during coronary artery occlusion in rats. Cytokine 47: 23-29, 2009.

17. Guo Z, Niu YL, Zhang JW and Yao TP: Coronary artery occlusion alters expression of substance $\mathrm{P}$ and its mRNA in spinal dorsal horn in rats. Neuroscience 145: 669-675, 2007.

18. Wang Q, He ZG, Li ZX, Li SY, Chen YL, Feng MH, Hong QX and Xiang HB: Bioinformatics analysis of gene expression profile data to screen key genes involved in cardiac ischemia-reperfusion injury. Int J Clin Exp Med 11: 4955-4966, 2018.

19. Trapnell C, Roberts A, Goff L, Pertea G, Kim D, Kelley DR, Pimentel H, Salzberg SL, Rinn JL and Pachter L: Differential gene and transcript expression analysis of RNA-seq experiments with TopHat and Cufflinks. Nat Protoc 7: 562-578 2012.

20. Trapnell C, Williams BA, Pertea G, Mortazavi A, Kwan G, van Baren MJ, Salzberg SL, Wold BJ and Pachter L: Transcript assembly and quantification by RNA-Seq reveals unannotated transcripts and isoform switching during cell differentiation. Nat Biotechnol 28: U511-U174, 2010.

21. Chen M, Li ZX, Wang Q and Xiang HB: Altered expression of differential genes in thoracic spinal cord involved in experimental cholestatic itch mouse model. Curr Med Sci 38: 679-683, 2018.

22. Wang Q, Li ZX, Liu BW, He ZG, Liu C, Chen M, Liu SG, Wu WZ and Xiang HB: Altered expression of differential gene and lncRNA in the lower thoracic spinal cord on different time courses of experimental obstructive jaundice model accompanied with altered peripheral nociception in rats. Oncotarget 8: 106098-106112, 2017.

23. Liu QQ, Liu H, He ZG, Zhang SJ, Liu BW, Wang L, Qiu WH, Xu Q, Xiang HB and Lv YM. Differential gene and lncRNA expression in the lower thoracic spinal cord following ischemia/ reperfusion-induced acute kidney injury in rats. Oncotarget 8:53465-53481, 2017.

24. Wang QL, Ai HZ, Liu JL, Xu M, Zhou Z, Qian C, Xie Y and Yan J: Characterization of novel lnc RNAs in the spinal cord of rats with lumbar disc herniation. J Pain Res 12: 501-512, 2019.

25. National Research Council (US) Institute for Laboratory Animal Research. In: Guide for the Care and Use of Laboratory Animals. National Academies Press (US), Washington, DC, 1996. 
26. Wang Q, Li ZX, Li YJ, Manyande A, Li SY, Feng MH, Wu DZ and Xiang HB: Alterations in amino acid levels and metabolite ratio of spinal cord in rat with myocardial ischemia-reperfusion injury by proton magnetic resonance spectroscopy. Am J Transl Res 11: 3101-3108, 2019.

27. Wang Q, Li ZX, Li YJ, He ZG, Chen YL, Feng MH, Li SY, Wu DZ and Xiang HB: Identification of IncRNA and mRNA expression profiles in rat spinal cords at various timepoints following cardiac ischemia/reperfusion. Int J Mol Med 43: 2361-2375, 2019

28. Pan XC, Li ZX, Wu DZ, Li SY, Xiang HB and Song YT: Mapping changes of whole brain blood flow in rats with myocardial ischemia/reperfusion injury assessed by positron emission tomography. Curr Med Sci 39: 653-657, 2019.

29. Li SY, Li ZX, He ZG, Wang Q, Li YJ, Yang Q, Wu DZ, Zeng HL and Xiang HB: Quantitative proteomics reveal the alterations in the spinal cord after myocardial ischemia-reperfusion injury in rats. Int J Mol Med 43: 1877-1887, 2019.

30. Xu Z, Alloush J, Beck E and Weisleder N: A murine model of myocardial ischemia-reperfusion injury through ligation of the left anterior descending artery. J Vis Exp 10: 51329, 2014

31. Liu S, Yang Y, Song YQ, Geng J and Chen QL: Protective effects of N(2)LalanylLglutamine mediated by the JAK2/STAT3 signaling pathway on myocardial ischemia reperfusion. Mol Med Rep 17: 5102-5108, 2018.

32. Liu BW, Li ZX, He ZG, Wang Q, Liu C, Zhang XW, Yang H and Xiang HB: Altered expression of itch-related mediators in the lower cervical spinal cord in mouse models of two types of chronic itch. Int J Mol Med 44: 835-846, 2019.

33. Liu QQ, Liu H, He ZG, Zhang SJ, Liu BW, Wang L, Qiu WH, Xu Q, Xiang HB and Lv YM: Differential gene and lncRNA expression in the lower thoracic spinal cord following ischemia/reperfusion-induced acute kidney injury in rats. Oncotarget 8: 53465-53481, 2017.

34. Pan N, Bhatti MZ, Zhang H, Ni B, Fan X and Chen J: The encystment-related micrornas and its regulation molecular mechanism in pseudourostyla cristata revealed by high throughput small RNA sequencing. Int J Mol Sci 21: 2309, 2020.

35. Sun H, Wang J, Que J, Peng Y, Yu Y, Wang L, Ye H, Huang K, Xue Y, Zhou Y and Ji K: RNA sequencing revealing the role of AMP-activated protein kinase signaling in mice myocardial ischemia reperfusion injury. Gene 703: 91-101, 2019.

36. Ke C, Gao F, Tian X, Li C, Shi D, He W and Tian Y: Slit2/Robo1 mediation of synaptic plasticity contributes to bone cancer pain Mol Neurobiol 54: 295-307, 2017.

37. Liu BW, Li ZX, He ZG, Liu C, Xiong J and Xiang HB: Altered expression of target genes of spinal cord in different itch models compared with capsaicin assessed by RT-qPCR validation. Oncotarget 8: 74423-74433, 2017.

38. Fu Q, Shi D, Zhou Y, Zheng H, Xiang H, Tian X, Gao F, Manyande A, Cao F, Tian Y and Ye D: MHC-I promotes apoptosis of GABAergic interneurons in the spinal dorsal horn and contributes to cancer induced bone pain. Exp Neurol 286: 12-20, 2016.

39. Guan XH, Fu QC, Shi D, Bu HL, Song ZP, Xiong BR, Shu B, Xiang HB, Xu B, Manyande A, et al: Activation of spinal chemokine receptor CXCR3 mediates bone cancer pain through an Akt-ERK crosstalk pathway in rats. Exp Neurol 263: 39-49, 2015

40. Xu B, Guan XH, Yu JX, Lv J, Zhang HX, Fu QC, Xiang HB, $\mathrm{Bu}$ HL, Shi D, Shu B, et al: Activation of spinal phosphatidylinositol 3-kinase/protein kinase B mediates pain behavior induced by plantar incision in mice. Exp Neurol 255: 71-82, 2014.

41. Livak KJ and Schmittgen TD: Analysis of relative gene expression data using real-time quantitative PCR and the 2(-Delta Delta C(T)) method. Methods 25: 402-408, 2001

42. Huang C, Wang J, Wang N, Du F, Xiong W, Qian J, Zhong K, Cai A, Xu S, Huang J, et al: Effect of myocardial ischemic preconditioning on ischemia-reperfusion stimulation-induced activation in rat thoracic spinal cord with functional MRI. Int J Cardiol 285: 59-64, 2019

43. Rajendran PS, Nakamura K, Ajijola OA, Vaseghi M, Armour JA, Ardell JL and Shivkumar K: Myocardial infarction induces structural and functional remodelling of the intrinsic cardiac nervous system. J Physiol 594: 321-341, 2016.

44. Huang WA, Boyle NG and Vaseghi M: Cardiac innervation and the autonomic nervous system in sudden cardiac death. Card Electrophysiol Clin 9: 665-679, 2017.

45. Dae MW, Lee RJ, Ursell PC, Chin MC, Stillson CA and Moise NS: Heterogeneous sympathetic innervation in German shepherd dogs with inherited ventricular arrhythmia and sudden cardiac death. Circulation 96: 1337-1342, 1997.
46. Stramba-Badiale M, Lazzarotti M and Schwartz PJ: Development of cardiac innervation, ventricular fibrillation, and sudden infant death syndrome. Am J Physiol 263: H1514-H1522, 1992.

47. Schwartz PJ: Cardiac sympathetic innervation and the prevention of sudden death. Cardiologia 35: 51-54, 1990

48. Scalercio L, Vitter J and Elliott CE: Placement of a continuous stellate ganglion block for treatment of refractory ventricular fibrillation in the setting of known prinzmetal angina during pregnancy: A case report. A A Pract 12: 106-108, 2019.

49. Imran TF, Malapero R, Qavi AH, Hasan Z, de la Torre B, Patel YR, Yong RJ, Djousse L, Gaziano JM and Gerhard-Herman MD: Efficacy of spinal cord stimulation as an adjunct therapy for chronic refractory angina pectoris. Int J Cardiol 227: 535-542, 2017.

50. Dobias M, Michalek P, Neuzil P, Stritesky M and Johnston P: Interventional treatment of pain in refractory angina. A review. Biomed Pap Med Fac Univ Palacky Olomouc Czech Repub 158: 518-527, 2014.

51. Deer TR, Mekhail N, Provenzano D, Pope J, Krames E, Thomson S, Raso L, Burton A, DeAndres J, Buchser E, et al: The appropriate use of neurostimulation of the spinal cord and peripheral nervous system for the treatment of chronic pain and ischemic diseases: The neuromodulation appropriateness consensus committee. Neuromodulation 17: 515-550, 2014.

52. Sylvén C: Neurophysiological aspects of angina pectoris. Z Kardiol 86: 95-105, 1997.

53. Pan X, Bao H, Si Y, Xu C, Chen H, Gao X, Xie X, Xu Y, Sun F and Zeng L: Spinal cord stimulation for refractory angina pectoris: A systematic review and meta-analysis. Clin J Pain 33: 543-551, 2017.

54. Hombach V, Grebe O, Merkle N, Waldenmaier S, Höher M, Kochs M, Wöhrle J and Kestler HA: Sequelae of acute myocardial infarction regarding cardiac structure and function and their prognostic significance as assessed by magnetic resonance imaging. Eur Heart J 26: 549-557, 2005.

55. Bär FW, Tzivoni D, Dirksen MT, Fernández-Ortiz A, Heyndrickx GR, Brachmann J, Reiber JH, Avasthy N, Tatsuno J, Davies M, et al: Results of the first clinical study of adjunctive CAldaret (MCC-135) in patients undergoing primary percutaneous coronary intervention for ST-elevation myocardial infarction: The randomized multicentre CASTEMI study. Eur Heart J 27: 2516-2523, 2006

56. Hearse DJ, Humphrey SM and Chain EB: Abrupt reoxygenation of the anoxic potassium-arrested perfused rat heart: A study of myocardial enzyme release. J Mol Cell Cardiol 5: 395-407, 1973.

57. Herzog WR, Vogel RA, Schlossberg ML, Edenbaum LR, Scott HJ and Serebruany VL: Short-Term low dose intracoronary diltiazem administered at the onset of reperfusion reduces myocardial infarct size. Int J Cardiol 59: 21-27, 1997.

58. De Stefani D, Raffaello A, Teardo E, Szabò I and Rizzuto R: A forty-kilodalton protein of the inner membrane is the mitochondrial calcium uniporter. Nature 476: 336-340, 2011.

59. Hausenloy DJ and Yellon DM: The mitochondrial permeability transition pore: Its fundamental role in mediating cell death during ischaemia and reperfusion. J Mol Cell Cardiol 35: 339-341, 2003.

60. Argaud L, Gateau-Roesch O, Muntean D, Chalabreysse L, Loufouat J, Robert D and Ovize M: Specific inhibition of the mitochondrial permeability transition prevents lethal reperfusion injury. J Mol Cell Cardiol 38: 367-374, 2005.

61. Pang KC, Frith MC and Mattick JS: Rapid evolution of noncoding RNAs: Lack of conservation does not mean lack of function. Trends Genet 22: 1-5, 2006.

62. Wang P, Luo ML, Song E, Zhou Z, Ma T, Wang J, Jia N, Wang G, Nie S, Liu Y and Hou F: Long noncoding RNA lnc-TSI inhibits renal fibrogenesis by negatively regulating the TGF-beta/smad3 pathway. Sci Transl Med 10: 2018.

63. Bejerano G, Pheasant M, Makunin I, Stephen S, Kent WJ, Mattick JS and Haussler D: Ultraconserved elements in the human genome. Science 304: 1321-1325, 2004.

64. Li D and Yang MQ: Identification and characterization of conserved lncRNAs in human and rat brain. BMC Bioinformatics 18: 489, 2017.

65. Sun Y, Fan W, Xue R, Dong B, Liang Z, Chen C, Li J, Wang Y, Zhao J, Huang H, et al: Transcribed ultraconserved regions, Uc.323, ameliorates cardiac hypertrophy by regulating the transcription of CPT1b (Carnitine Palmitoyl transferase 1b). Hypertension 75: 79-90, 2020.

This work is licensed under a Creative Commons Attribution-NonCommercial-NoDerivatives 4.0 International (CC BY-NC-ND 4.0) License. 